\title{
The clinical features of unstable haemoglobin disease
}

\author{
J. M. WHITE
}

From the Department of Haematology, Royal Postgraduate Medical School, London

The unstable haemoglobins now comprise the largest group of haemoglobin variants which result in red cell haemolysis in heterozygous carriers. At least 49 variants have been described in many parts of the world and in most ethnic groups. They are inherited as autosomal codominants and all but two have been found in heterozygous combination with $\mathrm{Hb} \mathrm{A}$, the exceptions being $\mathrm{Hb}$ Peterborough/Hb Lepore (King, Wiltshire, Lehmann, and Morinoto, 1972) and $\mathrm{Hb}$ Duarte $/ \beta$ thal (Beutler, Lang, and Lehmann, 1974). (For a list of the known unstable haemoglobins readers are referred to White, 1974.)

The molecular pathology of these variants has been the subject of at least three reviews (Carrell and Lehmann, 1969; White and Dacie, 1971); White, 1974), thus this report will consider mainly the clinical aspects, namely, the presentation, the laboratory diagnosis, and the treatment of affected patients.

Briefly, the molecular pathology of these variants is that the tertiary configuration of the abnormal polypeptide chain $(\alpha$ or $\beta$ ) is inherently unstable. This is caused by a single change in either the size or charge of an amino acid side chain, or else the deletion of the amino acid, in critical regions of the tightly coiled chain. This results in its unfolding, allowing water to enter the hydrophobic centre. The ensuing sequence of events is poorly understood in terms of protein chemistry. However, several oxidative states are recognized, and what is important is that the changes are irreversible. Rapid denaturation of the chain takes place, resulting eventually in its precipitation to Heinz bodies. In the steady state, continual denaturation takes place, a process which is greatly enhanced if the cells are exposed to oxidative drugs. It appears that the Heinz bodies are continually removed by the reticuloendothelial system, together with some membrane. Eventually a critical haemolytic state is reached when the cell is sequestered and phagocytosed. It should be added that the earlier evidence indicating chemical membrane damage due to Heinz body early attachment (Jacob, Brain, and Dacie, 1968) has not been confirmed (Winterbourn and Carrell, 1974).

The degree of instability amongst the variants shows a wide variation and consequently the clinical severity covers a wide spectrum from severe hae- molysis detectable shortly after birth to a very minor disorder discovered only on routine examination.

\section{Clinical Presentation}

The majority of patients have presented with an acute haemolytic crisis caused by oxidative drugs or infection. At these times they are usually cyanosed (sulphaemoglobinaemia), pale and jaundiced, and excrete very dark urine. Haemoglobinaemia and haemoglobinuria have been reported. The haematological indices are of little value in making the diagnosis as is the peripheral blood morphology which may be indistinguishable from that of favism. It should be stressed that there is no urgency in making the diagnosis at this stage. Moreover, since the results of various tests may be confusing, it is better to wait until the patient has recovered.

When patients present with chronic haemolytic anaemia the features, both clinical and haematological, vary enormously. If the haemolysis is intense the patients are often cyanosed and excrete dark (or even black) urine due to dipyrroluria. They are usually anaemic and have very high reticulocyte

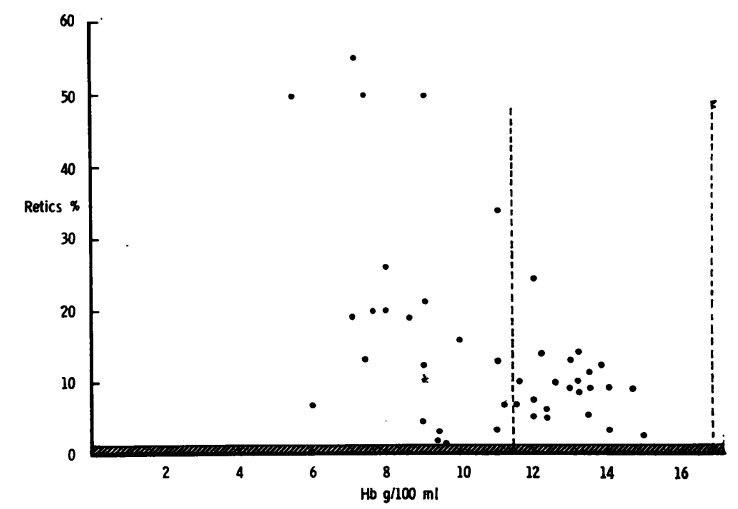

Fig 1 Reported values of haemoglobin levels and reticulocyte counts associated with 43 different unstable haemoglobin variants. Note that more than half of the variants are not associated with anaemia, probably due to increases in the oxygen affinity curve. With all, however, the reticulocyte count is increased. 
counts. The peripheral blood morphology depends largely on whether the spleen has been removed or not. Before splenectomy the picture is that of a chronic non-spherocytic haemolytic anaemia, although occasional contracted cells are seen. Basophilic stippling may be prominent. After splenectomy many irregular contracted cells are visible, often containing large, pale blue intracellular inclusions (Heinz bodies). In patients with only a moderate or a mild degree of haemolysis all of these features are less noticeable. They may not be cyanosed and the urine may be only slightly darker than normal. Many are not anaemic (fig 1) although in all the reticulocyte count is raised. The peripheral blood may not be strikingly abnormal even if the spleen has been removed; however, a number of small contracted cells is usually present.

\section{Approach to Diagnosis}

The unstable haemoglobinopathies are one of the oxidative haemolytic syndromes which includes drug-induced haemolysis and deficiencies in the hexose monophosphate shunt. Consequently, many clinical and haematological features are shared. There are, however, some laboratory findings associated with unstable haemoglobins which are specific in making the diagnosis.

The history is of little value except one of haemolysis after drugs, which indicates an increased susceptibility of the red cell to be oxidized. The belief that pigmenturia, although once thought to be specific for the unstable haemoglobinopathies, does not appear to be true. Patients with chronic haemolysis due to deficiencies in the hexose monophosphate shunt also appear to have dipyrroluria.

The peripheral blood film may or may not be of value and may not be distinguishable from that in any other non-spherocytic haemolytic anaemia. However, attention should be paid to the presence of small contracted cells and red cells in which the haemoglobin is not evenly distributed throughout the cell. Although these cells do not contain stainable Heinz bodies, the haemoglobin molecules are probably undergoing 'polymerization' before precipitation. Very few, if any, fragmented or 'drug-eaten' cells are seen. This is important since it indicates that the membrane is not being oxidized and that the oxidative stress is within the erythrocyte.

In establishing the diagnosis the approach should be logical, initially by carrying out screening tests, followed by more specific tests. The screening tests only indicate that an oxidative process is going on and the most useful are the levels of met- and sulphaemoglobin; the standard autohaemolysis test; and the glutathione (GSH) stability test (Beutler,
1957). The levels of met- and sulphaemoglobin are variable, and indeed may not be increased. The autohaemolysis test, however, is more useful, not in so far as indicating the extent of the haemolysis and whether or not it is corrected by glucose, but because of the formation of methaemoglobin and the generation of Heinz bodies. With normal cells, the formation of methaemoglobin is usually less than $5 \%$ and Heinz bodies are not normally seen. Even in cells which show a high degree of lysis, eg, pyruvate kinase deficiency, the formation of methaemoglobin is less than $10 \%$. However, in cells which carry an unstable haemoglobin or have a deficiency in the reducing system, the formation of methaemoglobin is usually greater than $20 \%$ and Heinz bodies are usually obvious. The GSH stability test is extremely useful although not specific. The dye test (Motulsky, Krant, Thieme, and Musto, 1959) and the Jacob and Jandl screening test (Jacob and Jandl, 1966) for G6PD deficiency are negative with cells containing an unstable haemoglobin. Haemoglobin electrophoresis, $\mathrm{Hb} F$ and $\mathbf{H b} \mathbf{A}_{2}$ estimations are extremely useful. About half of the unstable haemoglobins migrate with $\mathrm{Hb} \mathrm{A}$. However, many of them show, on electrophoresis, minor components migrating in the position of $\mathrm{Hb} \mathrm{S}$ or just anodally to $\mathbf{H b} \mathbf{A}_{2}$. These are haem-depleted components of the unstable haemoglobin, having the formula $\alpha_{2}{ }^{\mathrm{H}} \beta_{2}{ }^{\circ}$. Free $\alpha$ chains can then be seen migrating cathodally. The question of the use of organic solvents in preparing haemolysates is still unsettled. Carrell (unpublished data) has shown that chloroform does not result in any marked precipitation and loss of the unstable haemoglobin, but we have found that it results in precipitation of the free $\alpha$ chains, thus its use is to be avoided. The level of $\mathrm{Hb} \mathrm{A}_{2}$ in most of the $\beta$ chain unstable haemoglobins has been found to be increased to between 4 and $5 \%$. This is extremely valuable, since raised levels are not found in other oxidative haemolytic states where the stress falls on all intracellular haemoglobin components. Where measured, the $\mathrm{Hb} \mathrm{F}$ levels are generally increased, indicting a congenital haemolytic state.

The heat instability test is specific for unstable haemoglobin haemolytic anaemia in the steady state. Negative results are found in other causes of chronic oxidative haemolysis. However, false positives can be found in acute oxidative haemolytic crises, eg, favism. Although the test originally employed a $1: 25$ dilution of haemolysate in $0.1 \mathrm{~mol} / 1$ phosphate buffer, pH 7.4 (Grimes and Meisler, 1962), superior results are found by using $0 \cdot 1 \mathrm{~mol} / 1$ Tris $\mathrm{HCl}$ buffer (fig 2). The temperature should not exceed $50^{\circ} \mathrm{C}$, since several haemoglobin variants are unstable at 60 or $70^{\circ} \mathrm{C}$, the clinical significance 


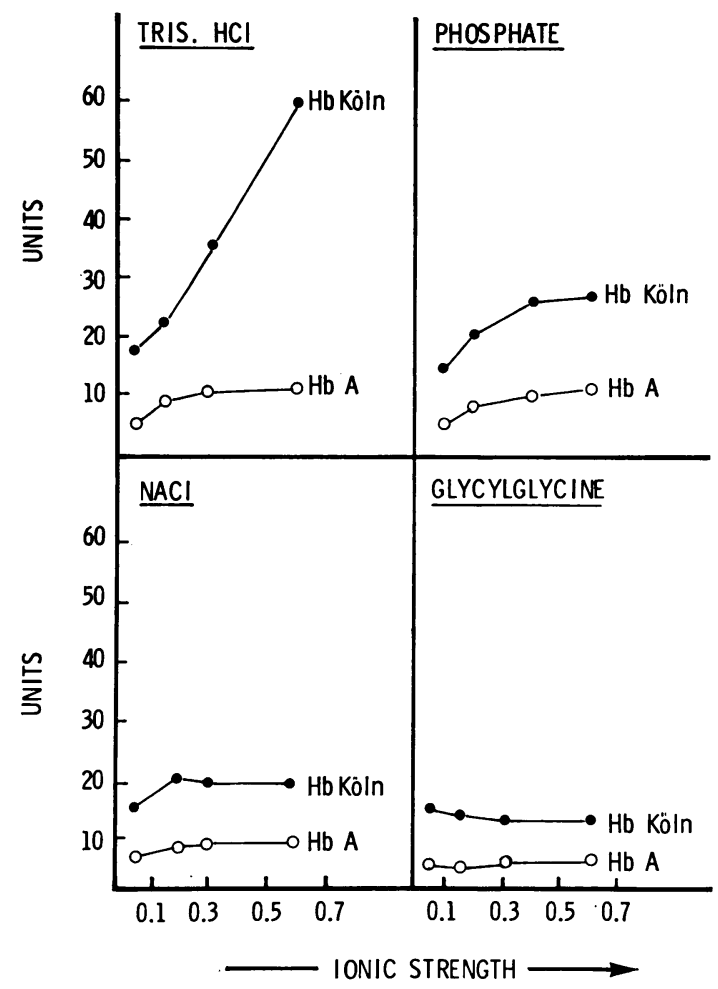

Fig 2 The effect of different diluents on the relative rates of precipitation of $\mathrm{Hb} K \ddot{\mathrm{oln}}$ and $\mathrm{Hb} A$. The rate of precipitation is expressed as units (I unit $=0 \cdot 1$ 280 difference in optical density/min). The samples were heated at $50^{\circ} \mathrm{C}$ and the increase in turbidity recorded by a Unicam S.P. 800 spectrophotometer. Note that $0 \cdot 1$ mol/l Tris $\mathrm{HCl}$ results in a more rapid precipitation of the unstable haemoglobin.

of which is doubtful. In our experience false positive results are more common than false negative results. However, if the amount of precipitable $\mathrm{Hb}$ is less than $5 \%$ it is of doubtful significance. The test must be controlled with a blood sample of the same age. Difficulty is often met in quantitating the percentage precipitation if the difference in optical density at $540 \mathrm{~nm}$ between the heated and unheated samples is used; more reliable results are obtained by measuring this at $280 \mathrm{~nm}$.

\section{Treatment}

The treatment of affected carriers largely depends on the severity of the patient's symptoms which have to be carefully assessed. Many variants show a wide degree of clinical expression within one family.
Also the same variant shows different expression in different families.

Splenectomy always appears to be of value (fig 3) and is probably essential in patients with severe haemolysis and anaemia. However, it is now realized that some variants associated with anaemia have reduced oxygen affinities, eg, $\mathrm{Hb}$ Hammersmith and $\mathrm{Hb}$ Seattle (Bellingham and Huehns, 1968). Although this never affords complete compensation when haemolysis is severe, in that most patients also have an increased cardiac output, it has to be taken into consideration. In contrast, many variants have an increased oxygen affinity, eg, $\mathrm{Hb}$ Köln, and though such patients have high haemoglobin levels (13-14 $\mathrm{g} / \mathrm{dl})$ they may be symptomatic and can benefit from splenectomy. In this last group, often supranormal haemoglobin levels can be found after splenectomy and cases of deep vein thrombosis and pulmonary embolism have been reported (Beutler, Lang, and Lehmann, 1974). This hazard has also to be taken into consideration when assessing the benefits to be gained by splenectomy.

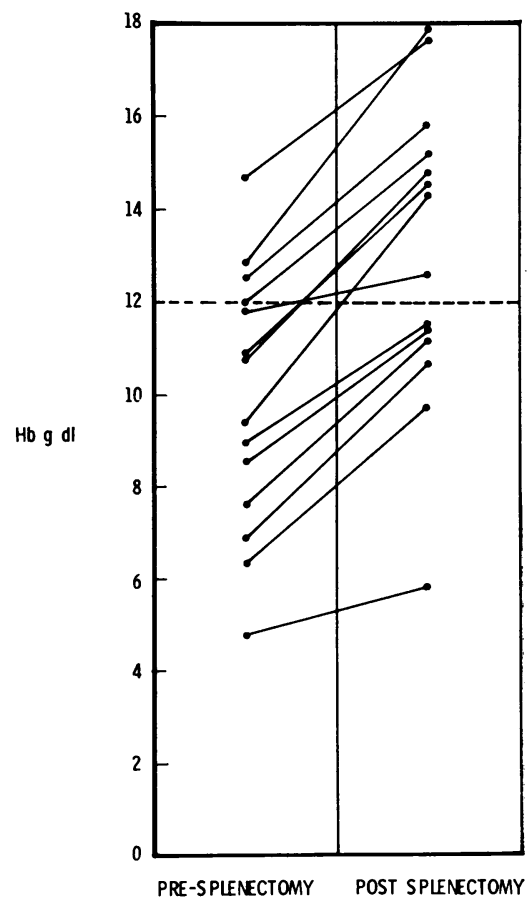

Fig 3 The effect of splenectomy on the level of haemoglobin in affected carriers (14 different variants). In all patients the level of haemoglobin increases. Note that before splenectomy some of the patients had normal haemoglobin levels and these rose to supranormal levels after splenectomy. 
All patients, once diagnosed, should avoid oxidative drugs but especially sulphonamides.

\section{Complications}

The most serious complication appears to be acute exacerbation of haemolysis following drugs or infection, but only one death has so far been reported. As in all chronic haemolytic states, gallstones are relatively common and the condition worsens during pregnancy. Overall, however, as a group of disorders, the disease is relatively benign and associated with longevity. There are only one or two patients heterozygous for highly unstable haemoglobins in whom the life span will probably be shortened.

\section{References}

Bellingham, A. J., and Huehns, E. R. (1968). Compensation in haemolytic anaemias caused by abnormal haemoglobins. Nature (Lond.), 218, 924-926.

Beutler, E. (1957). The glutathione instability of drug sensitive red cells. A new method for the in vitro detection of drug sensitivity. J. Lab clin, 49, 84-95.

Beutler, E., Lang, A., and Lehmann, H. (1974). Hemoglobin Duarte: $\left(a_{2} \beta_{2} 62\right.$ (E6) Ala $\rightarrow$ Pro): a new unstable hemoglobin with increased oxygen affinity. Blood, 43, 527-535.

Carrell, R. W., and Lehmann, H. (1969). The unstable haemoglobin haemolytic anaemias. Sem. Hemat., 6, 116-132.

Grimes, A. J., and Meisler, A. (1962). Possible cause of Heinz bodies in congenital Heinz-body anaemia. Nature (Lond.), 194, 190-191.

Jacob, H. S., Brain, M. C., and Dacie, J. V. (1968). Altered sulf hydryl reactivity of hemoglobins and red blood cell membranes in congenital Heinz body hemolytic anemia. J. clin. Invest., 47, 2664-2677.

Jacob, H. S., and Jandl, J. H. (1966). A simple visual screening test for glucose-6-phosphate dehydrogenase deficiency employing ascorbate and cyanide. New Engl. J. Med., 274, 1162-1167.

King, M. A. R., Wiltshire, B. G., Lehmann, H., and Morimoto, $H$. (1972). An unstable haemoglobin with reduced oxygen affinity: haemoglobin Peterborough $\beta 111$ (G 13) Valine $\rightarrow$ Phenylalanine, its interaction with normal haemoglobins and haemoglobin Lepore. Brit. J. Haemat., 22, 125-134.

Motulsky, A. G., Kraut, J. M., Thieme, W. T., and Musto, D. F. (1959). Biochemical genetics of glucose-6-phosphate dehydrogenase deficiency (Abstr.). Clin. Res., 7, 89-90.

White, J. M. (1974). The unstable haemoglobin disorders. Clin. Haemat., 3, 333-356.

White, J. M., and Dacie, J. V. (1971). The unstable hemoglobins: molecular and clinical features. Progr. Hemat., 7, 69-109.

Winterbourn, C. C., and Carrell, R. W. (1974). Studies of hemoglobin denaturation and Heinz body formation in the unstable hemoglobins. J. clin Invest., in press. 